\author{
Diana Marcela Montoya** \\ Carmen Dussán Lubert*** \\ Javier Taborda Chaurra**** \\ Luz Stella Nieto Osorio****
}

enero - junio / 18

3

in

82

ป ส

蛋

울

pedagógicas que inciden en el aprendizaje de estudiantes de la Universidad de Caldas según su estilo cognitivo", inscrita en la Vicerrectoría de Investigaciones y Postgrados de la Universidad de Caldas.

** Psicóloga. Magíster en Educación con énfasis en Relaciones Pedagógicas. Magíster en Neuropsicología. Docente Programa de Psicología y Especialización en Neuropsicopedagogía, Universidad de Manizales. Docente Departamento de Estudios Educativos, Universidad de Caldas. Grupo de Investigación en Cognición y Educación. Correspondencia: diana.montoya@ucaldas.edu.co ORCID: 0000-0001-8007-0102

*** Ingeniera Química. Magíster en Enseñanza de las Matemáticas. Docente Departamento de Matemáticas, Universidad de Caldas. Grupo de Investigación en Estadística y Matemáticas. Correspondencia: carmen.dussan@ucaldas.edu.co ORCID: 0000-0002-8093-6487

**** Licenciado en Educación: Educación Física. Especialista en Métodos de Enseńanza y Entrenamiento. Magíster en Educación y Desarrollo Humano. Doctor en Ciencias de la Educación. Docente Departamento de Estudios Educativos, Universidad de Caldas. Grupo de Investigación Currículum e identidades culturales. Correspondencia: javier.taborda@ucaldas.edu.co ORCID: 0000-0001-5917-5778

***** Licenciada en Educación Física. Especialista en Lúdica y Recreación para el desarrollo social y cultural. Magíster en Educación y Desarrollo Humano. Magíster en dirección de proyectos de Ocio, Cultura, Turismo, Deporte y Recreación. Doctora en Ocio y Desarrollo Humano. Docente del Departamento de Acción Física Humana, Universidad de Caldas. Grupo de Investigación Currículum e identidades culturales. Correspondencia: luz.nieto@ucaldas.edu.co ORCID: 0000-0003-1437-8664 


\section{Motivación y estrategias de aprendizaje en estudiantes de la Universidad de Caldas*}

Cómo citar este artículo: Montoya, D. M., Dussán Lubert, C., Taborda Chaurra, J., \& Nieto Osorio, L. S. (2018). Motivación y estrategias de aprendizaje en estudiantes de la Universidad de Caldas. Tesis Psicológica, 13(1), 82-101. https://doi.org/10.37511/tesis.v13n1a6

\section{ABSTRACT}

This research describes the behavior of students of a Colombian university before a validated test from which you can measure motivational components and use of learning strategies in the subjects. The correlation of subcategories between a scale of motivation and a scale of learning strategies was analyzed in order to establish the type of associations that could happen. 547 students from different undergraduate programs of the University of Caldas, took the Questionnaire of Motivation and Learning Strategies (CMEA). Positive correlation was found between orientation to intrinsic goals with orientation to extrinsic goals, value of the task and self-efficacy for learning; orientation to extrinsic goals with task value, control beliefs, self-efficacy for learning and test anxiety; value of the task with self-efficacy for learning and control beliefs with anxiety before the exams. All the items of the learning strategies scale (less the regulation of effort) are correlated in a positive way, effort regulation was not associated to the learning with elaboration. likewise, when the items of the motivation scale and the learning strategies were crossed, the associations were direct in most cases once again, except for control beliefs against organization and control beliefs against peer learning.

Keywords: motivation, cognitive strategies, metacognitive strategies, context strategies, self-regulated learning.

\author{
Recibido: 10 marzo 2018 \\ Revisado: 15 marzo 2018 \\ Aprobado: 28 junio 2018
}

\section{RESUMEN}

La presente investigación describe el comportamiento de estudiantes de una universidad colombiana ante una prueba validada a partir de la cual se pueden apreciar componentes motivacionales y uso de estrategias de aprendizaje en los sujetos. Se analizó la correlación de subcategorías entre una escala de motivación y una escala de estrategias de aprendizaje a fin de establecer el tipo de asociaciones que pudieran darse. Participaron en la investigación 547 estudiantes de diferentes programas de pregrado de la Universidad de Caldas, a los cuales se les aplicó el Cuestionario de Motivación y Estrategias de Aprendizaje (CMEA). Se encontró correlación positiva entre orientación a metas intrínsecas con orientación a metas extrínsecas, valor de la tarea y autoeficacia para el aprendizaje; orientación a metas extrínsecas con valor de la tarea, creencias de control, autoeficacia para el aprendizaje y ansiedad ante los exámenes; valor de la tarea con autoeficacia para el aprendizaje y creencias de control con ansiedad ante los exámenes. Todos los ítems de la escala de estrategias de aprendizaje (menos la regulación del esfuerzo) se correlacionan entre ellos de manera positiva, la regulación del esfuerzo no se asoció al aprendizaje con elaboración. Así mismo, cuando se cruzaron los ítems de la escala de motivación y de las estrategias de aprendizaje, nuevamente las asociaciones fueron directas en la mayoría de los casos, excepto para creencias de control contra organización y creencias de control contra aprendizaje con compañeros.

Palabras clave: motivación, estrategias cognitivas, estrategias metacognitivas, estrategias de contexto, aprendizaje autorregulado. 


\section{Introducción}

Frente a la nueva ciencia del aprendizaje se han considerado como campos emergentes de investigación la metacognición y el aprendizaje autorregulado (Sawyer, 2014, Ayala-Peña, 2015, Winne \& Azevedo, 2014), por cuanto se reconoce su aporte en el desarrollo de la capacidad de agencia del estudiante en relación con su propio proceso de aprendizaje (Balconi, 2010).

De esta manera puede indicarse, que la metacognición es entendida como el conocimiento y la cognición que las personas tienen sobre los objetos y productos cognitivos, es decir, sobre cualquier cosa cognitiva o psicológica (Flavell, 1979); es así como desde el origen del constructo, se asume que cualquier intento de monitoreo y supervisión de los procesos cognitivos puede ser considerado como una forma de metacognición (Flavell, 1987). En este sentido, Kuhn (2000) señala que la principal distinción entre cognición y metacognicion es que la segunda es considerada como una cognición de segundo orden (Buratti \& Allwood, 2015; Tarricone, 2011) en cuanto representa una especie de metanivel (Nelson \& Narens, 1990; Nelson, 1992).

De manera más reciente, el término es usado para referirse a la clase de cognición que puede ser consciente o inconsciente, y que se ocupa del desarrollo de la capacidad de agencia de la persona, sobre su propia actividad mental de manera consciente y deliberada. En tal sentido, se ha señalado que la metacognición se refleja en el conocimiento, la regulación y la consciencia que la persona establece acerca de sus procesos cognitivos, así como en relación con sus propios procesos de monitoreo y de control de la propia actividad cognitiva, denominada por algunos autores como cognición de primer orden (Flavel, 1987; Kuhn, 2000; Tarricone, 2011; Schraw \& Gutiérrez, 2015; Buratti \& Allwood, 2015).
En general, puede indicarse que la mayoría de modelos teóricos en metacognición están constituidos por componentes, por lo que algunos autores los han clasificado en modelos declarativos y procedimentales, con un marcado énfasis en el conocimiento y la regulación de la actividad cognitiva, con escasa consideración de las emociones y la motivación (Peña-Ayala \& Cárdenas, 2015).

El aprendizaje autorregulado representa un constructo relacionado con el actuar metacognitivo; sin embargo, presenta algunas diferencias. Para algunos investigadores, el aprendizaje autorregulado incluye además de los aspectos cognitivos y metacognitivos, los aspectos conductuales, motivacionales, emocionales y afectivos que estan implicados en el aprendizaje (Panadero, 2017), por lo cual representa una categoria que parece involucrar más variables en el proceso de regulación que solo los aspectos cognitivos y metacognitivos. Por lo mismo, dichos modelos de aprendizaje autorregulado más que constituirse por componentes, se estructuran por fases de aprendizaje, que dan lugar a un carácter ciclico y dinámico en el que multiples varibles se consideran en el aprendizaje (Azevedo, 2009).

En los estudios sobre metacognición, autorregulación y motivación se establece que el actuar metacognitivo requiere control deliberado, así se estima que la motivación juega un rol importante en el monitoreo metacognitivo efectivo. En este tipo de estudios se abordan los autorreportes de los aprendices acerca de su motivación para el aprendizaje, y entre los elementos que se analizan se consideran las características de la conducta y las habilidades metacognitivas (Pierce \& Lang, 2000; Pintrich, 2004; Suárez \& Fernández, 2011; Suárez, Fernández, Sánchez \& Zamora, 2016). En general, como alternativa conceptual en este tipo de estudios, se ha aplicado el modelo de Pintrich \& de 
Groot (1990) al estudio de la metacognición y la autorregulación del aprendizaje, considerando variables como la motivación intrínseca, la orientación al objetivo (valoración de la tarea), el control de las creencias en torno al aprendizaje y la autoeficacia, que respectivamente implican la regulación metacognitiva y la regulación del esfuerzo (Pintrich, Smith, García \& McKeachie, 1993).

A su vez, puede indicarse que los estudios sobre metacognición, aprendizaje autorregulado y el uso de estrategias metacognitivas se enfocan en el abordaje de las dificultades que encuentra el estudiante para autorregular su propio proceso de aprendizaje, entre las que se establecen: la forma cómo los estudiantes monitorean características de la cognición que engañan a su propio ejercicio de control metacognitivo tales como la posibilidad de revisar o de prestar atención a una tarea (Koriat, 1997; Winne \& Azevedo, 2014). Los estudios realizados desde esta perspectiva señalan que el aprendizaje autorregulado productivo depende del autocontrol, la autoeficacia, la autoestima y la seguridad (Koriat, 1997; Koriat \& Bjork, 2006; Koriat, Maayan, \& Nussinson, 2006; Dunlosky \& Rawson, 2012a). En esta perspectiva, se ha indicado que una de las características más importantes de los estudiantes que se autorregulan es que estos tienen estrategias de aprendizaje para lograr sus metas y monitorear la efectividad de estas estrategias (Winne \& Azevedo, 2014). Se requiere continuar avanzando en la realización de estudios que aborden el problema de la autorregulación del aprendizaje desde el análisis de componentes como la motivación y el uso de estrategias (cognitivas, metacognitivas y de contexto) en cuanto se considera que los aprendices autorregulados son participantes activos en su proceso de aprendizaje, establecen metas, seleccionan y aplican estrategias y son capaces de controlar su propia efectividad (Zimmerman, 2008).
$\mathrm{Al}$ respecto se ha señalado que algunas de las características comunes de los aprendices autorregulados permiten plantear que estos estudiantes son conscientes y reconocen la relación entre el proceso de autorregulación y el éxito académico, conocen cuáles son sus capacidades, sus conocimientos y las estrategias de aprendizaje que mejor les permiten aprender; así mismo, monitorean (supervisan) y controlan (regulan) el uso de diversos tipos de estrategias cognitivas, metacognitivas, motivacionales y de apoyo relacionadas con el contexto, el ambiente y la inversión de tiempo y esfuerzo (Monereo, 2001; Núñez, González-Pienda \& Rosario, 2006; Nocito, 2013).

Desde esta perspectiva, se requiere que los estudiantes desarrollen procesos metacognitivos y de autorregulación de su aprendizaje cada vez más eficientes a fin de que puedan demostrar en su desempeño diario que se conocen, se regulan y se hacen progresivamente más conscientes de su propia responsabilidad en relación con su proceso de aprendizaje. Así se ha considerado que los estudiantes hacen una regulación efectiva de su aprendizaje cuando ellos pueden determinar precisamente qué conocen y qué no conocen alrededor de contenidos de dominio (Gutiérrez, 2013). Este proceso de autoconocimiento acerca de la propia eficacia permite que los aprendices focalicen su atención y sus demás recursos cognitivos en lo que realmente les hace falta aprender, realizando procesos de monitoreo, control y ajuste sobre el objetivo y valor de la tarea, el plan de acción para conseguirla que implica la asignación de tiempo, así como la elección del tipo de estrategias de aprendizaje a emplear-, además de la evaluación y la autoreflexión que el estudiante realice en el curso de la ejecución y una vez finalizada la tarea.

En relación con el objetivo de la presente investigación, se consideran antecedentes importantes un estudio desarrollado por Ramírez (2016)

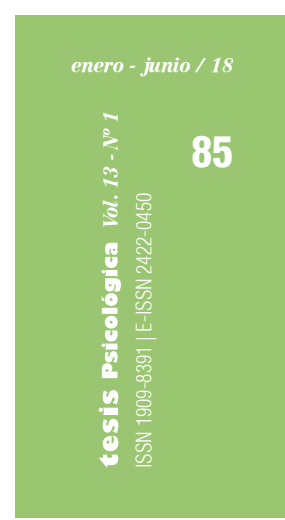


en el cual fue aplicada la versión en español (CMEA) del Motivated Strategies for Learning Questionnaire (MSQL), en una muestra de 1140 estudiantes $(531$ hombres y 609 mujeres) de nueve programas académicos de pregrado, que dio cuenta de diferencias significativas por género en esta escala a favor de las mujeres y "correlaciones positivas significativas entre los factores motivacionales y de uso de estrategias de aprendizaje y la edad y el curso" (p. 30). Haciendo uso del mismo cuestionario, Granados \& Gallego (2016), con una muestra de 593 estudiantes (196 hombres y 397 mujeres) de tres universidades, de diferentes programas académicos mostraron la "existencia relacional entre la implementación de las estrategias de aprendizaje de tipo cognitivo así como la implementación de estrategias de autorregulación" (p. 77).

En el presente artículo se presentan los resultados de una investigación, que tuvo como objetivo caracterizar la motivación y el uso de estrategias cognitivas, metacognitivas y de contexto en una muestra de estudiantes universitarios de la ciudad de Manizales, en el segundo semestre de 2016.

\section{Metodología}

Diseño: investigación cuantitativa, de tipo descriptivo-correlacional de carácter transversal (Hernández, Fernández \& Baptista, 2010).

\section{Población objeto de estudio}

Se trabajó con estudiantes matriculados en 2016-I en la asignaturas a cargo de los docentes que participaron en la presente investigación, en la que se incluyeron los programas de pregrado en Biología, Desarrollo Familiar, Enfermería, Geología, Ingeniería de Alimentos, Ingeniería en Sistemas, Licenciatura en Artes Escénicas, Licenciatura en Biología y Química,
Licenciatura en Educación Física, Licenciatura en Lenguas Modernas y Medicina.

\section{Tipo de muestreo}

Muestra no probabilística de 547 estudiantes de la Universidad de Caldas, los cuales participaron voluntariamente de la investigación.

\section{Criterios de inclusión}

Los estudiantes tenían edades comprendidas entre los 16 y 30 ańos, no podían presentar alteraciones neurológicas ni psiquátricas; además, no debían presentar historia de repitencia, rezago o fracaso escolar. Por último, debían firmar el consentimiento informado. Los casos fueron seleccionados por un especialista en psiquiatría del equipo de investigación. La historia del proceso académico fue establecida desde el reporte que brinda al programa de la Oficina de Registro Académico de la Universidad, previa presentación del consentimiento informado del estudiante.

\section{Instrumento}

Se trabajó con la traducción al español del test desarrollado por Pintrich, Smith, García \& McKeachie (1991), en la versión adaptada y validada del Motivated Strategies for Learning Questionnarie (MSLQ) por Ramírez, Canto, Bueno \& Echazarreta (2013), bajo el nombre Cuestionario de Motivación y Estrategias de Aprendizaje (CMEA).

El Motivated Strategies for Learning Questionnarie es un instrumento de medida de autorreporte de 81 reactivos que mide el uso de estrategias de aprendizaje y el nivel de motivación de los estudiantes. El test incluye aspectos de la autorregulación del aprendizaje desde la perspectiva de la autorregulación metacognitiva, a partir de la cual se reconoce la 
relación entre motivación y cognición (Schunk \& Zimmerman, 1994; Zimmerman \& Schunk, 1989; Zimmerman, 1989; Pintrich, Smith, Garcia \& McKeachine, 1993). El MSLQ fue desarrollado desde el paradigma cognitivo-social de la motivación y de las estrategias de aprendizaje (García \& Pintrich, 1991). La escala de motivación consta de 31 ítems distribuidos en 6 subescalas que miden: las metas, las creencias de valor, el control de pensamientos, las creencias acerca de las habilidades para tener éxito y la ansiedad ante los exámenes. La escala de estrategias de aprendizaje incluye 31 ítems relativos al conocimiento y uso que los estudiantes hacen de diferentes estrategias cognitivas y metacognitivas, así como el manejo de recursos para el aprendizaje por parte del estudiante. En la validación al español del cmea se confirmó la estructura factorial original y la consistencia interna del MSLQ con 6 subescalas de motivación y 9 de estrategias de aprendizaje (Ramírez, Canto, Bueno \& Echazarreta, 2013).

\section{Variables utilizadas en la investigación}

Además del género y la edad del entrevistado, se trabajó con los ítems del instrumento MSLQ/ CMEA, los cuales se muestran en la tabla 1 .

\section{TABLA 1. Ítems del instrumento MSLQ}

\begin{tabular}{|c|c|c|c|}
\hline Escala & Subescala & Variable & Código \\
\hline \multirow{6}{*}{$\begin{array}{l}\text { Escala de } \\
\text { motivación }\end{array}$} & \multirow{3}{*}{$\begin{array}{l}\text { Componentes } \\
\text { de valor }\end{array}$} & $\begin{array}{l}\text { Orientación a } \\
\text { metas intrínsecas }\end{array}$ & omi \\
\hline & & $\begin{array}{l}\text { Orientación } \\
\text { a metas } \\
\text { extrínsecas }\end{array}$ & ome \\
\hline & & Valor de la tarea & vt \\
\hline & \multirow{2}{*}{$\begin{array}{l}\text { Componentes } \\
\text { de expectativa }\end{array}$} & $\begin{array}{l}\text { Creencias de } \\
\text { control }\end{array}$ & $\mathrm{CC}$ \\
\hline & & $\begin{array}{l}\text { Autoeficacia para } \\
\text { el aprendizaje }\end{array}$ & aepa \\
\hline & $\begin{array}{c}\text { Componentes } \\
\text { afectivos }\end{array}$ & $\begin{array}{l}\text { Ansiedad ante los } \\
\text { exámenes }\end{array}$ & ae \\
\hline
\end{tabular}

\begin{tabular}{|c|c|c|c|}
\hline Escala & Subescala & Variable & Código \\
\hline \multirow{4}{*}{$\begin{array}{l}\text { Estrategias de } \\
\text { aprendizaje }\end{array}$} & \multirow{4}{*}{$\begin{array}{c}\text { Estrategias } \\
\text { metacognitivas }\end{array}$} & Repetición & re \\
\hline & & Elaboración & ela \\
\hline & & Organización & org \\
\hline & & $\begin{array}{l}\text { Pensamiento } \\
\text { crítico }\end{array}$ & $\mathrm{pc}$ \\
\hline \multirow{5}{*}{$\begin{array}{l}\text { Estrategias de } \\
\text { aprendizaje }\end{array}$} & $\begin{array}{c}\text { Estrategias } \\
\text { metacognitivas }\end{array}$ & $\begin{array}{c}\text { Autorregulación } \\
\text { metacognitiva }\end{array}$ & arm \\
\hline & \multirow{4}{*}{$\begin{array}{l}\text { Estrategias } \\
\text { de manejo de } \\
\text { recursos }\end{array}$} & $\begin{array}{l}\text { Administración } \\
\text { del tiempo y del } \\
\text { ambiente }\end{array}$ & ata \\
\hline & & $\begin{array}{l}\text { Regulación del } \\
\text { esfuerzo }\end{array}$ & RegEsf \\
\hline & & $\begin{array}{l}\text { Aprendizaje con } \\
\text { compañeros }\end{array}$ & $\mathrm{ac}$ \\
\hline & & $\begin{array}{c}\text { Búsqueda de } \\
\text { ayuda }\end{array}$ & ba \\
\hline
\end{tabular}

Fuente: Autores

\section{Análisis estadístico}

Para comparar las medias entre géneros se prueba primero el cumplimiento del supuesto de normalidad de los datos mediante el test de KolmogorovSmirnov; si tal supuesto es válido se utiliza la $\mathrm{t}$ de Student para medias independientes. En caso que el supuesto no sea válido, se utiliza la prueba U de Mann-Whitney (Sheskin, 2007).

La correlación entre las variables estudiadas se analizó utilizando el coeficiente de correlación de Pearson (o el de Spearman), dependiendo de si los datos presentan o no una distribución normal (Daniel, 2002). Los análisis se realizaron utilizando el software SPSS versión 23.

\section{Resultados}

Se trabajó con 259 mujeres (47.3\%) y 288 hombres $(52.7 \%)$, cuyas edades promedio y desviaciones estándar fueron de 20.9 años y 2.7 años, para las mujeres, y de 22.1 años y 3.8 años para los hombres, la edad media de estos últimos fue mayor al interior de la universidad $(\mathrm{P}$ valor $=$ 0.0000). Contrasta esta aplicación del MSLQ con la descrita por Ramírez, Canto, Bueno \&

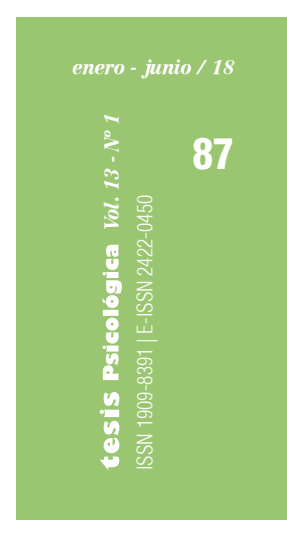


Echazarreta (2013), quienes en su origen pesaron su aplicación a estudiantes de un curso específico, pero los autores mencionados ampilaron el abanico de estudiantes y áreas $(\mathrm{N}=1140)$ y lo aplicaron a sujetos de ambos géneros (mujeres: $53.4 \%$ y hombres: $46.6 \%$ ), de diferentes titulaciones y cursos, al igual que se hace en el presente estudio. La media de edad para el estudio reportado fue de 20.89 años, con una desviación típica de 2.915, datos muy similares a los que reportamos en nuestro estudio. Vale la pena mencionar que la comparación que se hace en esos aspectos relaciona aplicaciones del MSLQ adaptado como cmea en tanto este último es más cercano a nuestro contexto que el MSLQ, más propio para aplicación en contextos anglosajones.

Se concuerda con Ramírez et ál. (2013) en que una de las razones para desarrollar este tipo de indagaciones con estudiantes universitarios se

TABLA 2. Estadísticos de la escala de motivación asocia a que "es en este nivel educativo en el que se produce un mayor control de su motivación y de sus estrategias cognitivas, metacognitivas y de contexto para la obtención de un nivel de logro educativo aceptable" (p. 200).

\section{Escala de motivación}

De acuerdo con la tabla 2, los hombres y mujeres tienden a obtener las mismas medias en orientación a metas intrínsecas, valor de la tarea y autoeficacia para el aprendizaje, pero difieren en orientación a metas extrínsecas y ansiedad ante los exámenes, donde ellas obtienen mayor promedio. En el caso de la variable creencias de control, los hombres presentan un mejor desempeño que sus compañeras. El coeficiente de variación señala que la ansiedad ante los exámenes es la variable que muestra mayor variabilidad o dispersión en sus medidas, especialmente para los estudiantes hombres.

\begin{tabular}{cccccccc} 
Género & Estadístico & OMI & OME & VT & CC & AEPA & AE \\
\hline \multirow{3}{*}{ Mujeres } & Media & 5.3 & 5.3 & 5.9 & 4.8 & 5.6 & 4.7 \\
\cline { 2 - 8 } & Desv. Est. & 1.0 & 1.2 & 1.0 & 1.0 & 0.9 & 1.2 \\
\cline { 2 - 8 } & Coef. Var. & $19.7 \%$ & $23.0 \%$ & $16.9 \%$ & $20.1 \%$ & $16.2 \%$ & $26.5 \%$ \\
\hline \multirow{3}{*}{ Hombres } & Media & 5.3 & 5.1 & 5.8 & 5.0 & 5.7 & 4.2 \\
\cline { 2 - 8 } & Desv. Est. & 1.0 & 1.2 & 1.0 & 0.9 & 0.8 & 1.3 \\
\cline { 2 - 8 } & Coef. Var. & $19.4 \%$ & $23.5 \%$ & $16.6 \%$ & $18.3 \%$ & $14.5 \%$ & $32.1 \%$ \\
\hline \multirow{3}{*}{ Universidad } & Media & 5.3 & 5.2 & 5.8 & 4.9 & 5.6 & 4.4 \\
\cline { 2 - 8 } & Desv. Est. & 1.0 & 1.2 & 1.0 & 0.9 & 0.9 & 1.3 \\
\cline { 2 - 8 } & Coef. Var. & $19.6 \%$ & $23.4 \%$ & $16.9 \%$ & $19.3 \%$ & $15.4 \%$ & $29.8 \%$ \\
\hline
\end{tabular}

Fuente: Autores

Lo señalado en el párrafo anterior se confirmó al realizar la prueba t de Student o la U de ManWhitney - dependiendo de si la variable se comportaba de manera normal o no-, se encontraron diferencias significativas entre hombres y mujeres (mayor media para las mujeres) en las variables: orientación a metas extrínsecas, ansiedad ante los exámenes y creencias de control (P valores de $0.0247 ; 0.0000$ y 0.0334 , respectivamente).
La matriz de correlación de Pearson o Spearman, dependiendo de la normalidad de las variables entre temas de la escala de motivación (tabla 3) indicó que los siguientes ítems se correlacionan de manera directa - cuando un ítem se incrementa, el otro también lo hace-:

- Orientación a metas intrínsecas con orientación a metas extrínsecas, valor de la tarea y autoeficacia para el aprendizaje. 
- Orientación a metas extrínsecas con valor de la tarea, creencias de control, autoeficacia para el aprendizaje y ansiedad ante los exámenes.

- Valor de la tarea con autoeficacia para el aprendizaje.

- Creencias de control con ansiedad ante los exámenes.

- La autoeficacia para el aprendizaje se asocia inversamente con ansiedad ante los exámenes.

TABLA 3. Correlación entre ítems de la escala de motivación

\begin{tabular}{cccccc} 
Variable & OME & VT & CC & AEPA & AE \\
\hline OMI & $\mathbf{0 . 0 0 1 8}$ & $\mathbf{0 . 0 0 0 0}$ & 0.1904 & $\mathbf{0 . 0 0 0 0}$ & 0.3382 \\
\hline OME & & $\mathbf{0 . 0 0 0 0}$ & $\mathbf{0 . 0 0 0 7}$ & $\mathbf{0 . 0 0 0 0}$ & $\mathbf{0 . 0 0 0 0}$ \\
\hline VT & & & 0.0923 & $\mathbf{0 . 0 0 0 0}$ & 0.5913 \\
\hline CC & & & & 0.5631 & $\mathbf{0 . 0 0 0 0}$ \\
\hline AEPA & & & & & $\mathbf{0 . 0 0 0 5}$
\end{tabular}

Fuente: Autores

Nota: $P$ valores significantes en negrilla.
La tabla 4 muestra la correlación entre subescalas de la motivación, discriminadas por género. Se observó asociación de carácter lineal y directo (en negrilla) entre la mayoría de ítems evaluados, con excepción para las mujeres en:

- Ansiedad ante los exámenes con orientación a metas intrínsecas, valor de la tarea y autoeficacia para el aprendizaje.

- Autoeficacia para el aprendizaje con creencias de control.

Para los hombres se encontraron menos asociaciones entre ítems. Las creencias de control solo se asocian de manera directa con ansiedad ante los exámenes y esta última se correlaciona también directamente con orientación a metas intrínsecas y de manera inversa con ansiedad ante los exámenes.

TABLA 4. Correlación entre subescalas de motivación para hombres y mujeres de la Universidad de Caldas

\begin{tabular}{ccccccccccc}
\multirow{2}{*}{ Subescala } & \multicolumn{4}{c}{ Mujeres } \\
\cline { 2 - 12 } & OME & VT & CC & AEPA & AE & OME & VT & CC & AEPA & AE \\
\hline OMI & $\mathbf{0 . 0 4 2 6}$ & $\mathbf{0 . 0 0 0 0}$ & $\mathbf{0 . 0 3 6 6}$ & $\mathbf{0 . 0 0 0 0}$ & 0.9788 & $\mathbf{0 . 0 2 9 0}$ & $\mathbf{0 . 0 0 0 0}$ & 0.6402 & $\mathbf{0 . 0 0 0 0}$ & 0.1523 \\
\hline OME & & $\mathbf{0 . 0 0 0 0}$ & $\mathbf{0 . 0 0 0 9}$ & $\mathbf{0 . 0 0 0 0}$ & $\mathbf{0 . 0 0 0 0}$ & & $\mathbf{0 . 0 0 0 4}$ & 0.1089 & $\mathbf{0 . 0 0 0 0}$ & $\mathbf{0 . 0 0 0 0}$ \\
\hline VT & & & $\mathbf{0 . 0 3 4 6}$ & $\mathbf{0 . 0 0 0 0}$ & 0.5141 & & & 0.8232 & $\mathbf{0 . 0 0 0 0}$ & 0.6494 \\
\hline CC & & & 0.4763 & $\mathbf{0 . 0 0 0 0}$ & & & & 0.6697 & $\mathbf{0 . 0 0 0 0}$ \\
\hline AEPA & & & & 0.0954 & & & & & $\mathbf{0 . 0 0 3 2}$ \\
\hline
\end{tabular}

Fuente: Autores

Nota: $P$ valores significantes en negrilla.

Estrategias de aprendizaje: se aprecia que la mayoría de las medias de los ítems de estrategias de aprendizaje en las estudiantes mujeres tienden a ser superiores que las de sus compañeros hombres (tabla 5), ellos se muestran más homogéneos en la mayoría de las variables.

TABLA 5. Estadísticos de estrategias de aprendizaje para las estudiantes mujeres de la Universidad de Caldas

\begin{tabular}{ccccccccccc} 
Género & Estadístico & RE & ELA & ORG & PC & ARI & ATA & RegEsf & AC & BA \\
\hline \multirow{3}{*}{ Mujeres } & Media & 4.9 & 4.9 & 5.2 & 4.9 & 4.8 & 4.8 & 4.4 & 4.3 & 5.2 \\
\cline { 2 - 10 } & $\begin{array}{c}\text { Desviación } \\
\text { estándar }\end{array}$ & 1.2 & 1.2 & 1.3 & 1.2 & 0.9 & 0.8 & 0.9 & 1.4 & 1.0 \\
\cline { 2 - 10 } & Coef. De Variac. & $25.1 \%$ & $24.4 \%$ & $24.9 \%$ & $24.5 \%$ & $18.3 \%$ & $15.9 \%$ & $21.4 \%$ & $33.0 \%$ & $20.2 \%$ \\
\hline
\end{tabular}




\begin{tabular}{|c|c|c|c|c|c|c|c|c|c|c|}
\hline Género & Estadístico & RE & ELA & ORG & PC & ARM & ATA & RegEsf & AC & BA \\
\hline \multirow{3}{*}{ Hombres } & Media & 4.5 & 4.8 & 4.5 & 4.9 & 4.6 & 4,7 & 4.4 & 4.2 & 5.0 \\
\hline & $\begin{array}{l}\text { Desviación } \\
\text { estándar }\end{array}$ & 1.1 & 1.1 & 1.2 & 1.1 & 0.8 & 0.6 & 0.9 & 1.4 & 1.0 \\
\hline & Coef. De Variac. & $25.2 \%$ & $22.8 \%$ & $27.2 \%$ & $22.4 \%$ & $17.2 \%$ & $13.2 \%$ & $19.6 \%$ & $34.0 \%$ & $20.8 \%$ \\
\hline \multirow{3}{*}{ Universidad } & Media & 4.7 & 4.9 & 4.8 & 4.9 & 4.7 & 4.7 & 4.4 & 4.2 & 5.1 \\
\hline & $\begin{array}{l}\text { Desviación } \\
\text { estándar }\end{array}$ & 1.2 & 1.1 & 1.3 & 1.1 & 0.8 & 0.7 & 0.9 & 1.4 & 1.1 \\
\hline & Coef. De Variac. & $25.5 \%$ & $23.6 \%$ & $27.0 \%$ & $23.4 \%$ & $17.9 \%$ & $14.6 \%$ & $20.5 \%$ & $33.5 \%$ & $20.6 \%$ \\
\hline
\end{tabular}

Fuente: Autores

Al realizar pruebas de hipótesis para comparar las medias entre hombres y mujeres de la Universidad de Caldas, se encontraron diferencias significativas entre géneros para repetición, organización y autorregulación metacognitiva, que fueron superiores en todos los casos para las mujeres (P valores de 0,$0000 ; 0,0000$ y 0,0044 , respectivamente).
La matriz de correlación indica asociaciones de carácter lineal resaltadas con negrilla, las cuales son todas directas (tabla 6); únicamente la regulación del esfuerzo no se asocia a aprendizaje con elaboración.

TABLA 6. Matriz de correlación entre estrategias de aprendizaje

\begin{tabular}{ccccccccc} 
Variable & ELA & ORG & PC & ARM & ATA & RegEsf & AC & BA \\
\hline RE & $\mathbf{0 . 0 0 0 0}$ & $\mathbf{0 . 0 0 0 0}$ & $\mathbf{0 . 0 0 0 0}$ & $\mathbf{0 . 0 0 0 0}$ & $\mathbf{0 . 0 0 0 0}$ & $\mathbf{0 . 0 0 0 0}$ & $\mathbf{0 . 0 0 0 0}$ & $\mathbf{0 . 0 0 0 0}$ \\
\hline ELA & & $\mathbf{0 . 0 0 0 0}$ & $\mathbf{0 . 0 0 0 0}$ & $\mathbf{0 . 0 0 0 0}$ & $\mathbf{0 . 0 0 0 0}$ & 0.0558 & $\mathbf{0 . 0 0 0 0}$ & $\mathbf{0 . 0 0 0 0}$ \\
\hline ORG & & & $\mathbf{0 . 0 0 0 0}$ & $\mathbf{0 . 0 0 0 0}$ & $\mathbf{0 . 0 0 0 0}$ & $\mathbf{0 . 0 1 9 9}$ & $\mathbf{0 . 0 0 0 0}$ & $\mathbf{0 . 0 0 0 0}$ \\
\hline PC & & & $\mathbf{0 . 0 0 0 0}$ & $\mathbf{0 . 0 0 0 0}$ & $\mathbf{0 . 0 0 0 3}$ & $\mathbf{0 . 0 0 0 0}$ & $\mathbf{0 . 0 0 0 0}$ \\
\hline ARM & & & & $\mathbf{0 . 0 0 0 0}$ & $\mathbf{0 . 0 0 0 0}$ & $\mathbf{0 . 0 0 0 0}$ & $\mathbf{0 . 0 0 0 0}$ \\
\hline ATA & & & & $\mathbf{0 . 0 0 0 0}$ & $\mathbf{0 . 0 0 0 0}$ & $\mathbf{0 . 0 0 0 0}$ \\
\hline RegEs & & & & & & $\mathbf{0 . 0 0 0 0}$ & $\mathbf{0 . 0 0 0 0}$ \\
\hline AC & & & & & & & & $\mathbf{0 . 0 0 0 0}$
\end{tabular}

Al discriminar por géneros (tablas 7 y 8), nuevamente se aprecia tanto para hombres como para mujeres, que todas las correlaciones son positivas - cuando se incrementa el valor en una variable se incrementa también el valor de la variable con la que se compara-. Para las mujeres todos los ítems se correlacionan; mientras que para los hombres la regulación del esfuerzo no se asocia con la elaboración ni la organización ni con el pensamiento crítico. 
TABLA 7. Matriz de correlación entre estrategias de aprendizaje para estudiantes mujeres

\begin{tabular}{|c|c|c|c|c|c|c|c|c|}
\hline Variable & ELA & ORG & PC & ARM & ATA & RegEsf́ & AC & BA \\
\hline RE & 0.0000 & 0.0000 & 0.0000 & 0.0000 & 0.0000 & 0.0009 & 0.0000 & 0.0000 \\
\hline ELA & & 0.0000 & 0.0000 & 0.0000 & 0.0000 & 0.0302 & 0.0000 & 0.0000 \\
\hline ORG & & & 0.0000 & 0.0000 & 0.0000 & 0.0174 & 0.0000 & 0.0000 \\
\hline PC & & & & 0.0000 & 0.0000 & 0.0014 & 0.0000 & 0.0000 \\
\hline ARM & & & & & 0.0000 & 0.0000 & 0.0000 & 0.0000 \\
\hline ATA & & & & & & 0.0000 & 0.0000 & 0.0000 \\
\hline RegEsf & & & & & & & 0.0000 & 0.0000 \\
\hline$A C$ & & & & & & & & 0.0000 \\
\hline
\end{tabular}

Fuente: Autores

Nota: $P$ valores significantes en negrilla.

TABLA 8. Matriz de correlación entre estrategias de aprendizaje para estudiantes hombres

\begin{tabular}{|c|c|c|c|c|c|c|c|c|}
\hline Variable & ELA & ORG & PC & ARM & ATA & RegEsf & AC & BA \\
\hline RE & 0.0000 & 0.0000 & 0.0000 & 0.0000 & 0.0000 & 0.0084 & 0.0000 & 0.0000 \\
\hline ELA & & 0.0000 & 0.0000 & 0.0000 & 0.0000 & 0.7140 & 0.0000 & 0.0000 \\
\hline ORG & & & 0.0000 & 0.0000 & 0.0000 & 0.2589 & 0.0000 & 0.0000 \\
\hline PC & & & & 0.0000 & 0.0000 & 0.0847 & 0.0000 & 0.0000 \\
\hline ARM & & & & & 0.0000 & 0.0000 & 0.0000 & 0.0000 \\
\hline ATA & & & & & & 0.0002 & 0.0000 & 0.0000 \\
\hline RegEsf & & & & & & & 0.0000 & 0.0000 \\
\hline$A C$ & & & & & & & & 0.0000 \\
\hline
\end{tabular}

Fuente: Autores

Nota: $P$ valores significantes en negrilla.

Escalas de motivación y estrategias de aprendi- de creencias de control contra organización y zaje: al cruzar la escala de motivación y las estra- aprendizaje con compañeros. Las creencias de tegias de aprendizaje sin discriminar por género control y ansiedad ante los exámenes son los (tabla 9), se observan asociaciones de carácter ítems que menos asociaciones muestran con las positivo en la mayoría de las celdas, a excepción estrategias de aprendizaje.

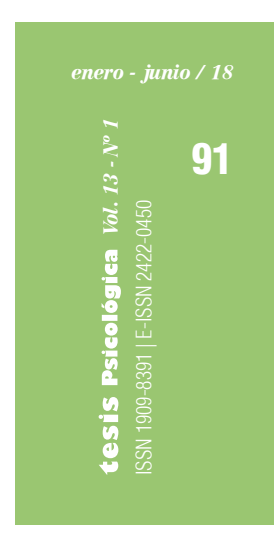

TABLA 9. Matriz de correlación entre escala de motivación y estrategias de aprendizaje

\begin{tabular}{ccccccc} 
Variables & ONI & ONE & VT & CC & AEPA & AE \\
\hline RE & $\mathbf{0 . 0 0 0 0}$ & $\mathbf{0 . 0 0 0 0}$ & $\mathbf{0 . 0 0 0 0}$ & 0.3779 & $\mathbf{0 . 0 0 0 0}$ & $\mathbf{0 . 0 0 0 0}$ \\
\hline ELA & $\mathbf{0 . 0 0 0 0}$ & $\mathbf{0 . 0 1 1 3}$ & $\mathbf{0 . 0 0 0 0}$ & 0.7784 & $\mathbf{0 . 0 0 0 0}$ & 0.3142 \\
\hline ORG & $\mathbf{0 . 0 0 0 0}$ & $\mathbf{0 . 0 0 0 0}$ & $\mathbf{0 . 0 0 0 0}$ & $\mathbf{0 . 0 0 0 6}$ & $\mathbf{0 . 0 0 0 0}$ & 0.9273 \\
\hline PC & $\mathbf{0 . 0 0 0 0}$ & 0.0510 & $\mathbf{0 . 0 0 0 0}$ & 0.7834 & $\mathbf{0 . 0 0 0 0}$ & 0.2090 \\
\hline ARM & $\mathbf{0 . 0 0 0 0}$ & $\mathbf{0 . 0 0 0 0}$ & $\mathbf{0 . 0 0 0 0}$ & 0.1729 & $\mathbf{0 . 0 0 0 0}$ & $\mathbf{0 . 0 0 0 9}$ \\
\hline ATA & $\mathbf{0 . 0 0 0 0}$ & $\mathbf{0 . 0 0 0 0}$ & $\mathbf{0 . 0 0 0 0}$ & 0.0705 & $\mathbf{0 . 0 0 0 0}$ & 0.0000 \\
\hline
\end{tabular}




\begin{tabular}{ccccccc} 
Variables & OMI & OME & VT & CC & AEPA & AE \\
\hline RegEsf & 0.1987 & $\mathbf{0 . 0 0 0 0}$ & 0.6740 & $\mathbf{0 . 0 0 0 1}$ & $\mathbf{0 . 0 4 1 1}$ & $\mathbf{0 . 0 0 0 0}$ \\
\hline AC & $\mathbf{0 . 0 0 0 0}$ & $\mathbf{0 . 0 0 0 0}$ & $\mathbf{0 . 0 0 0 0}$ & $\mathbf{0 . 0 2 7 0}$ & $\mathbf{0 . 0 0 0 0}$ & 0.3253 \\
\hline BA & $\mathbf{0 . 0 0 0 0}$ & $\mathbf{0 . 0 0 0 0}$ & $\mathbf{0 . 0 0 0 0}$ & 0.5856 & 0.0000 & 0.0004
\end{tabular}

Fuente: Autores

Nota: $P$ valores significantes en negrilla.

Al discriminar por géneros, todas las asociaciones en las mujeres son de carácter positivo. Las creencias de control y la ansiedad ante los exámenes son los ítems que menos correlaciones presentan con las estrategias de aprendizaje (tabla 10).
Para los hombres existe menor número de asociaciones, y de ellas: creencias de control y organización, y creencias de control y aprendizaje con compañeros son inversas (tabla 11).

TABLA 10. Matriz de correlación entre escala de motivación y estrategias de aprendizaje para las estudiantes mujeres

\begin{tabular}{ccccccc} 
Variables & OMII & OME & VT & CC & AEPA & AE \\
\hline RE & $\mathbf{0 . 0 0 0 0}$ & $\mathbf{0 . 0 0 0 0}$ & $\mathbf{0 . 0 0 0 0}$ & $\mathbf{0 . 0 1 0 2}$ & $\mathbf{0 . 0 0 0 0}$ & $\mathbf{0 . 0 0 1 8}$ \\
ELA & $\mathbf{0 . 0 0 0 0}$ & 0.0814 & $\mathbf{0 . 0 0 0 0}$ & 0.8336 & $\mathbf{0 . 0 0 0 0}$ & 0.2240 \\
\hline ORG & $\mathbf{0 . 0 0 0 0}$ & $\mathbf{0 . 0 0 4 5}$ & $\mathbf{0 . 0 0 0 0}$ & 0.6208 & $\mathbf{0 . 0 0 0 0}$ & 0.6641 \\
\hline PC & $\mathbf{0 . 0 0 0 0}$ & 0.3997 & $\mathbf{0 . 0 0 0 0}$ & 0.6629 & $\mathbf{0 . 0 0 0 0}$ & 0.5480 \\
\hline ARM & $\mathbf{0 . 0 0 0 0}$ & $\mathbf{0 . 0 0 0 0}$ & $\mathbf{0 . 0 0 0 0}$ & $\mathbf{0 . 0 2 6 1}$ & $\mathbf{0 . 0 0 0 0}$ & 0.1030 \\
\hline ATA & $\mathbf{0 . 0 0 0 0}$ & $\mathbf{0 . 0 0 0 0}$ & $\mathbf{0 . 0 0 0 0}$ & 0.0977 & $\mathbf{0 . 0 0 0 0}$ & $\mathbf{0 . 0 0 0 4}$ \\
\hline RegEsf & 0.0509 & $\mathbf{0 . 0 0 0 0}$ & 0.6548 & $\mathbf{0 . 0 0 3 9}$ & $\mathbf{0 . 0 3 8 3}$ & $\mathbf{0 . 0 0 0 0}$ \\
\hline AC & $\mathbf{0 . 0 0 0 0}$ & $\mathbf{0 . 0 0 0 0}$ & $\mathbf{0 . 0 0 0 0}$ & 0.3670 & $\mathbf{0 . 0 0 0 0}$ & 0.6771 \\
\hline BA & $\mathbf{0 . 0 0 0 0}$ & $\mathbf{0 . 0 0 0 4}$ & $\mathbf{0 . 0 0 0 0}$ & 0.5713 & $\mathbf{0 . 0 0 0 0}$ & $\mathbf{0 . 0 0 6 0}$ \\
\hline
\end{tabular}

Fuente: Autores

Nota: $P$ valores significantes en negrilla.

TABLA 11. Matriz de correlación entre escalas de motivación y estrategias de aprendizaje para los estudiantes hombres

\begin{tabular}{ccccccc} 
Variables & OMI & ONE & VT & CC & AEPA & AE \\
\hline RE & $\mathbf{0 . 0 0 0 0}$ & 0.0000 & 0.0000 & 0.3100 & $\mathbf{0 . 0 0 0 0}$ & 0.0741 \\
\hline ELA & $\mathbf{0 . 0 0 0 0}$ & 0.1194 & $\mathbf{0 . 0 0 0 0}$ & 0.5253 & $\mathbf{0 . 0 0 0 0}$ & 0.5116 \\
\hline ORG & $\mathbf{0 . 0 0 0 0}$ & $\mathbf{0 . 0 0 8 6}$ & $\mathbf{0 . 0 0 0 0}$ & 0.0001 & $\mathbf{0 . 0 0 0 0}$ & 0.2365 \\
\hline PC & $\mathbf{0 . 0 0 0 0}$ & 0.0528 & $\mathbf{0 . 0 0 0 0}$ & 0.7537 & $\mathbf{0 . 0 0 0 0}$ & 0.2862 \\
\hline ARM & $\mathbf{0 . 0 0 0 0}$ & $\mathbf{0 . 0 0 0 0}$ & $\mathbf{0 . 0 0 0 0}$ & 0.8672 & $\mathbf{0 . 0 0 0 0}$ & $\mathbf{0 . 0 1 9 2}$ \\
\hline ATA & $\mathbf{0 . 0 0 2 8}$ & $\mathbf{0 . 0 0 0 0}$ & $\mathbf{0 . 0 0 0 2}$ & 0.3638 & $\mathbf{0 . 0 0 0 5}$ & 0.0565 \\
\hline RegEsf & 0.7343 & $\mathbf{0 . 0 1 3 9}$ & 0.2109 & $\mathbf{0 . 0 0 7 8}$ & 0.6035 & $\mathbf{0 . 0 0 0 0}$ \\
\hline AC & $\mathbf{0 . 0 0 0 2}$ & $\mathbf{0 . 0 4 9 8}$ & $\mathbf{0 . 0 0 0 0}$ & $\mathbf{0 . 0 4 0 0}$ & $\mathbf{0 . 0 1 4 0}$ & 0.5520 \\
\hline BA & $\mathbf{0 . 0 0 6 2}$ & $\mathbf{0 . 0 0 0 0}$ & $\mathbf{0 . 0 0 0 1}$ & 0.8617 & 0.0002 & 0.0540
\end{tabular}

Fuente: Autores

Nota: $P$ valores significantes en negrilla. 


\section{Discusión}

El objetivo de este artículo se centró en dar cuenta de las características de los componentes motivacionales y el uso de estrategias metacognitivas en una muestra de estudiantes universitarios de la ciudad de Manizales, en el segundo semestre de 2016. Para hacerlo se atendieron tres frentes: la caracterización según género y edad en asociación con escalas de motivación y estrategias de aprendizaje de la muestra seleccionada; la presentación de caracteres especiales de los sujetos en las escalas de motivación y estrategias de aprendizaje, y por último, la correlación que se presentaba entre las dos escalas mencionadas. A continuación se presentan comparativos con la revisión realizada y se dará cuenta de algunos límites de la investigación y de perspectivas a futuro.

Una revisión a nuestros estadísticos para la escala de motivación muestra medias para hombres y mujeres muy similares para OMI, OME, VT, CC y AEPA. Una diferencia significativa se presenta en las medias de esta escala en ansiedad ante los exámenes (AE). Teniendo en cuenta que el rango de puntuación va de 1 (nada cierto en mí) a 7 (totalmente cierto en mí), en cada uno de los items el resultado tanto en hombres como en mujeres deja ver en motivación un nivel general de 5.2, un poco por encima de resultados obtenidos por Martín, Bueno \& Ramírez (2010), quienes en un trabajo realizado con estudiantes mexicanos obtuvieron una media en escala de motivación de 4.95. Resultado que hay que tomar con reserva en tanto se desarrolló en contextos de bachillerato y con estudiantes hombres y mujeres de edades promedio de 16.3 años. A pesar de ello, la diferencia a favor de la muestra del presente estudio podría estar relacionada con que en el presente estudio se trabajó con estudiantes de mayor edad y de contextos universitarios. En cuanto a los estudiantes universitarios, siguiendo a Ramírez, Canto, Bueno
\& Echazarreta (2013), puede indicarse que en esta población estudiantil "se produce un mayor control de su motivación y de sus estrategias cognitivas, metacognitivas y de contexto para la obtención de un nivel de logro educativo aceptable” (p. 200). Llama la atención en un estudio de Ramírez (2016) que "las mujeres obtiene puntuaciones mayores que los varones en casi la totalidad de las subescalas motivacionales" (p. 238); nuestro estudio muestra a favor de la mujer diferencia significativa en AE, cuestión que valdría la pena considerar a fondo en posteriores estudios en tanto, a modo de hipótesis, podría considerarse como un rasgo con mayor estabilidad en mujeres que en hombres.

Este asunto de la motivación en los estudiantes debe recordarse siguiendo a Rothman, Baldwin, Hertel \& Fuglestad (2011), pues ha sido históricamente entendido como un factor importante de la habilidad de los estudiantes para iniciar y mantener un patrón de conducta, esto es, una manifestación más o menos frecuente de acciones o comportamientos íntimamente vinculados a las solicitudes que los estudios universitarios le hacen a los sujetos.

Como se había ya expuesto en la matriz de correlación de la escala de motivación, se encontraron ítems que correlacionan directamente y de los cuales se pueden también encontrar referencias en la literatura. Como ejemplo, Granados \& Gallego (2016) reportan que en su investigación.

Las relaciones más fuertes resultaron para las subescalas de motivación en cuanto a: orientación a metas intrínsecas (OMI), la cual presentó una correlación con el valor de la tarea (VT) de 0.532 , y con la autoeficacia para el aprendizaje (AEPA) de 0.550. (p. 81).

El presente estudio mostró correlación directa entre orientación a metas intrínsecas, extrínsecas, valor de la tarea y autoeficacia para el

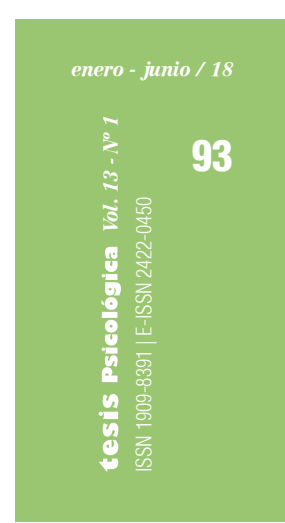


aprendizaje. Schunk (2012) apoya la correlación encontrada: "el hecho de adoptar una orientación a una meta de aprendizaje se asocia con una mayor autoeficacia, valor de la tarea y logro" (p. 431). Schunk, además, es partidario de apoyar la idea según la cual la actuación de la gente es coherente con la creencia de que dicha actuación puede ayudar a alcanzar metas y que para ello es necesario un cierto sentido de autoeficacia.

La correlación que se encontró entre orientación a metas extrínsecas con valor de la tarea, creencias de control, autoeficacia para el aprendizaje y ansiedad para los exámenes se debe tomar con prudencia entre otras cosas porque en un estudio reportado, la relación con la ansiedad en los exámenes no fue objeto de análisis y porque se consideraron correlaciones en dos actividades diferentes: académicas o escolares y deportivas. En sentido de lo aludido, en una investigación desarrollada por Duda y Nicholls (como se citó en Schunk, 2012) se puede apreciar que para deporte y trabajo escolar:

la orientación de la tarea se relacionaba con las creencias de alumnos de preparatoria de que el éxito depende del esfuerzo y la colaboración con los compañeros, mientras que la orientación al yo, se asociaba con las creencias de que el éxito se debe a una capacidad elevada y al intento por tener un mejor desempeño que los demás. La relación entre la orientación a la meta y las creencias acerca del éxito con la capacidad percibida no era fuerte. La capacidad percibida se relacionaba más con la satisfacción en el deporte que en la escuela, en tanto que para la orientación a la tarea se obtuvo el patrón opuesto (p. 396).

Lo expuesto plantea un reto a futuro en tanto algunas variables no vinculadas en las escalas de motivación y aprendizaje utilizadas en el presente estudio, que permitieron derivar la correlación mencionada, podrán complementarse con estudios acerca de la capacidad percibida y la satisfacción en la tarea, contrastando actividades en la universidad con otras prácticas extraescolares de los sujetos.

Sobre el asunto de las creencias de control, el planteamiento de Schunk (2012) es de interés en tanto el sentimiento o la creencia de control sobre las diferentes dimensiones de la tarea se relaciona de manera importante con diferentes dimensiones de la motivación. Esto significa en parte que si se puede tener control sobre las propias capacidades y las tareas, esto correlaciona bien con la motivación para triunfar. En tales circunstancias las creencias podrían correlacionar con la ansiedad ante las tareas y los exámenes tal como se muestra con los resultados de nuestro estudio. Debe considerarse también, siguiendo a Schunk (2012), que la creencia de competencia y de control interno se relaciona positivamente con una de las variables de la motivación: la motivación intrínseca, expresión que vuelve a colocar en primer plano las correlaciones directas que hemos encontrado en la matriz de correlación para la escala de motivación.

En el análisis de la escala de estrategias de aprendizaje nuestros datos mostraron puntuaciones similares, en escala de 1 a 7 entre hombres y mujeres para la regulación del esfuerzo (Reg.Esf/4,4) y para el pensamiento crítico $(\mathrm{PC} / 4,9)$. Hay amplia diferencia en RE a favor de las mujeres (4.9; hombres 4.5) y en ORG a favor también de las mujeres (5.2; hombres 4.5). En las otras variables como ELA, ARM y ATA, AC y BA los resultados son muy similares. En conjunto, el promedio para hombres y mujeres en esta escala es de 4.71, superior al del estudio de Martín, Bueno \& Ramírez (2010), que en la escala de estadísticos de aprendizaje para hombres y mujeres en población de bachilleres mexicanos reportaron una media de 4.53. La reserva mencionada en relación con la escala de motivación se renueva para estos resultados contrastados con nuestro estudio. 
Adicionalmente, de los resultados se ha destacado que sin discriminar por género, se presentan asociaciones directas, excepto entre los ítems de esfuerzo y de elaboración. Es decir que hay evidente asociación entre las demás variables como regulación del esfuerzo, repetición, organización, pensamiento crítico, autorregulación metacognitiva, adminstración del tiempo y el ambiente, y aprendizaje con compañeros. Tales asociaciones encajan con algunos de los hallazgos de Ramírez, Canto, Bueno \& Echazarreta (2013), quienes hicieron la traducción y validación psicométrica del MSLQ, denominado en español como CMEA, desde los cuales se ha indicado que

para que una habilidad académica llegue a ser ejecutada con maestría, los estudiantes deberán conductualmente, aplicar estrategias cognitivas a una tarea dentro de un contexto determinado. Para lograr esto se requiere de repetidos intentos de aprendizaje, ya que la maestría involucra la coordinación de los componentes personales, conductuales y contextuales o ambientales, cada uno de los cuales es dinámico por sí solo, pero lo son también cuando interactúan conjuntamente (p. 198).

Ahora bien, debe recordarse de nuestros datos que existen asociaciones positivas - $\mathrm{a}$ excepción de CC, ORG y AC- entre variables de las escalas de motivación y de estrategias de aprendizaje. Igualmente señalamos que las creencias de control y ansiedad ante los exámenes son los estadísticos de menor asociación con las diferentes subcategorías de las estrategias de aprendizaje.

Lo dicho se presenta en acuerdo con hallazgos de García \& Pintrich (1991) según los cuales hay correlaciones "muy altas" entre factores asociados a la escala de motivación — como AEPA y OMI-y el aprendizaje autorregulado asociado al tiempo, el esfuerzo, la metacognicón y la elaboración. Estas correlaciones muy altas, y las que en este estudio se denominan como positivas, tienen importante relación con lo que menciona Martín, Bueno \& Ramírez (2010) haciendo referencia a García \& Pintrich (1991), al señalar que: "la adopción de una orientación de aprendizaje y de dominio, y las evaluaciones positivas sobre la propia competencia llevan a una mayor utilización de las estrategias de control, la elaboración y el manejo del esfuerzo" (p.68). Siendo así, los resultados de este estudio, que reseñan estadísticos para las escalas de motivación y de estrategias de aprendizaje por encima de la media, rescatarían un potencial importante para el aprendizaje autorregulado de los estudiantes evaluados, lo que concuerda con hallazgos de un estudio ya clásico de Archer (1994) y que coincidiría con otros resultados reportados por Multon \& Brown (1991), estudios todos citados por Martin, Bueno \& Ramírez (2010).

Igualmente, los resultados de este estudio encajan con afirmaciones de Schunk (2012) según las cuales:

existe una relación estrecha ente la motivación y el aprendizaje, que se influyen mutuamente. La motivación de los estudiantes puede influir en lo que aprenden y en cómo lo aprenden. A su vez, a medida que los estudiantes aprenden y perciben que se vuelven cada vez más hábiles, se sienten motivados para seguir aprendiendo (p. 356).

\section{Conclusiones}

En momentos en que la Universidad de Caldas se encuentra orientando importantes esfuerzos para promover la promoción y la graduación con calidad de los estudiantes y para favorecer el desarrollo de la mejor formación de sus profesionales, la investigación desarrollada y la aplicación del MSLQ modificado como CMEA en el presente estudio son una alternativa viable para considerar la evaluación de relaciones importantes entre motivación y estrategias para 
el aprendizaje autónomo y autorregulado, y en consecuencia, diseñar procesos de enseñanza coherentes con estos resultados.

Lo indicado guarda relación con la necesidad de reconocer aportaciones de la llamada "nueva cultura del aprendizaje" en tanto, como se ha mencionado, la autorregulación de los aprendizajes, el control sobre los propios procesos cognitivos y la atención a diversas estrategias de aprendizaje, que correlacionarían muy bien con el rendimiento académico de los estudiantes, se encuentran en la agenda de atención de la mayor parte de las instituciones educativas del país. La aplicación de los docentes y de los aprendices sobre la habilidad para dar cuenta de los propios aprendizajes y de sus procesos de autoregulación pueden ser beneficiosos para muchos estudiantes (Dunlosky \& Rawson, 2012b). En parte, porque una educación de calidad asociada a apuestas por la formación y promoción con calidad de los estudiantes son hoy criterios importantes a tener encuentra en los procesos formativos y en indicadores para acreditación de alta calidad. Téngase en cuenta al respecto, por ejemplo, que la identificación de sujetos autorregulados o no autorregulados hace posible tomar decisiones en torno a estrategias de aprendizaje, enseñanza y didácticas que permitan la promoción de sus aprendizajes, cuestión por la cual los resultados de este estudio, en tanto orientador, pueden constituir un elemento importante para generar propuestas de atención al estudiantado, con el fin de que estos puedan hacer más eficientes sus aprendizajes.

En el contexto mencionado deben comprenderse los resultados, en lo fundamental destaca en la escala de motivación que los estudiantes hombres y mujeres tienden a obtener las mismas medias en orientación a metas intrínsecas, valor de la tarea y autoeficacia para el aprendizaje, pero difieren en orientación a metas extrínsecas y ansiedad ante los exámenes, donde ellas tienden a obtener mayores medias que sus compañeros hombres, y creencias de control, donde los hombres superan a las mujeres. Destacan también los resultados desde los cuales se evidencia que la ansiedad ante los exámenes es la variable que muestra mayor incertidumbre, especialmente en los estudiantes hombres. Además, la matriz de correlación para la escala de motivación (sin discriminar por géneros) permite señalar que los siguientes ítems se correlacionan de manera directa: orientación a metas intrínsecas con orientación a metas extrínsecas, valor de la tarea y autoeficacia para el aprendizaje; orientación a metas extrínsecas con valor de la tarea, creencias de control, autoeficacia para el aprendizaje y ansiedad ante los exámenes; valor de la tarea con autoeficacia para el aprendizaje, y creencias de control con ansiedad ante los exámenes. La autoeficacia para el aprendizaje se asoció inversamente con ansiedad ante los exámenes.

Sobre la escala de estrategias de aprendizaje en la que se consideraron variables como la elaboración, la organización, el pensamiento crítico, la autorregulación metacognitiva, la administración del tiempo y el ambiente, la regulación del esfuerzo, el aprendizaje con compañeros y la búsqueda de ayuda la matriz de correlación sin discriminar por género mostró asociaciones de carácter lineal, todas directas; únicamente la regulación del esfuerzo no se asoció al ítem de aprendizaje con elaboración.

Ahora bien, cuando se cruzaron la escala de motivación y las estrategias de aprendizaje sin discriminar por género, se observaron asociaciones de carácter positivo, a excepción de creencias de control contra organización y aprendizaje con compañeros. Las creencias de control y la ansiedad ante los exámenes son los ítems que menos asociaciones mostraron con las estrategias de aprendizaje. 
Los resultados señalan el importante vínculo que la misma teoría y diversos estudios han establecido, entre la presencia y el uso de diferentes estrategias de aprendizaje con el mantenimiento sostenido de la motivación de los estudiantes universitarios. Sin embargo, no deja de plantear un límite que en corto tiempo debe ser superado, relacionado con la ausencia o escasez hasta el momento de estudios que aborden estas variables con universitarios colombianos, que podrían representar grupos poblacionales con mayor similitud regional y contextual, y por lo mismo, con mayor validez ecológica, con los cuales poder establecer comparaciones, cuestión que abre un abanico a posibilidades de indagación, sumando variables como características de sueño, estilo cognitivo y autoconcepto, entre otros.

Una cuestión adicional sobre perspectivas a futuro del presente estudio tiene que ver con el vínculo que podría establecerse entre motivación, estrategias de aprendizaje y rendimiento académico que acá, tangencialmente, ha sido planteado y que no estaba en la finalidad del estudio, pero que en atención a múltiples referentes consultados venía apareciendo en la literatura. Como lo han mostrado García y Pintrich
(1991), en el aprendizaje autorregulado y su mantenimiento juegan un rol muy importante las variables asociadas a la motivación y su control. La medición de las escalas de autorregulación y de estrategias de aprendizaje según estos autores se asocia con el rendimiento académico. Estas relaciones se aprecian en otros contextos evaluados, pero en la Universidad de Caldas aún no han sido investigadas sobre todo por resultados que han sido ya establecidos en diferentes trabajos del grupo de investigación de Hederich, en los que se ha señalado la relación entre aprendizaje autorregulado y logro escolar (López, Hederich \& Camargo, 2012). Sería de interés plantear para una aproximación a lo dicho una hipótesis según la cual conocer los propios procesos de autorregulación hace posible alcanzar mejoras importantes en la autorregulación del aprendizaje, cuestión que nos pone al límite de análisis prometedores en relación con la autorregulación y el desempeño de los estudiantes, aunque como expresa Nocito (2013) "un funcionamiento cognitivo-motivacional eficaz suele ir asociado con aprendizajes altamente comprensivos y significativos, aunque ello no tiene por qué generar necesariamente buenos resultados académicos" (p. 164). 


\section{Referencias}

Archer, J. (1994). Achievements goals as a mesasure of motivation in university students. Contemporary Educational Psychology, 19, 430-446.

Azevedo, R. (2009).Theoretical, conceptual, methodological, and instructional issues in research on metacognition and self-regulated learning: A discussion. Metacognition Learning 4,87-95. Doi: 10.1007/s11409-009-9035-7

Ayala-Peña, A (Ed). (2015). Metacognition: fundaments, applications and trends. New York: Springer International. Balconi, M (2010). Neuropsychology of the sense of agency. Italia: Springer.

Daniel, W (2002). Bioestadística. Base para el análisis de las ciencias de la salud. México D.F: Limusa Wiley.

Duda, J \& Nicholls, J. (1992). Dimensions of achievement motivation in schoolwork and sport. Journal of Educational Psychology, 84, 290-299.

Dunlosky, J. \& Rawson, K. A. (2012a). Despite their promise, there's still a lot to learn about techniques that support durable learning. Journal of Applied Research in Memory and Cognition, 1(4), 254-256.

Dunlosky, J. \& Rawson, K. A. (2012b). Overconfidence produces underachievement: Inaccurate self evaluations undermine student's learning and retention. Journal Learning and Instruction, 22, 271-280.

Flavell, J. (1979). Metacognitive and Cognitive monitoring: a new area of cognitive developmental inquiry. American Psychology, 34(10), 906-911.

Flavell, J. (1987). Speculation about nature and development of metacognition. En F. Weinert, R. Kluwe. Metacognition, motivation and understanding. New Jersey: Hillsdale.

García, T., \& Pintrich P. (1991). Student motivation and self regulated learning: A LISREL model. Paper presented at the Annual meeting of the American Research Association, Chicago, IL.

Granados, H., \& Gallego, F. (2016). Motivación, aprendizaje autorregulado y estrategias de aprendizaje en estudiantes de tres universidades de Caldas y Risaralda. Revista Latinoamericana de Estudios Educativos, 12(1), 71-90. 
Gutiérrez, A (2013). Enhancing the calibration accuracy of adult learners: A multifaceted intervention. University of Nevada. Recuperado de http://digitalscholarship.unlv.edu/cgi/viewcontent. cgi? article $=2569$ \& context $=$ theses dissertations

Hernández, R., Fernández, C., \& Baptista, M (2010). Metodología de la investigación. México D.F.: McGraw-Hill. Koriat, A (1997). Monitoring one's own knowledge during study: A cue-utilization approach to judgments of learning. Journal of Experimental Psychology: General, 126(4), 349-370.

Koriat, A., \& Bjork, R. A. (2006). Illusions of competence during study can be remedied by manipulations that enhance learners' sensitivity to retrieval conditions at test. Memory \& Cognition, 34, 959-972.

Koriat, A., Maayan, H., \& Nussinson, R. (2006). The intricate relationships between monitoring and control in metacognition: Lessons for the cause-and-effect relation between subjective experience and behavior. Journal of Experimental Psychology: General, 135(1), 36-69.

Kuhn, D (2000). Metacognitive Development. American Psychological Society, 9(5), 178-181.

López, O., Hederich, C., \& Camargo, A. (2012). Logro en matemáticas, autorregulación del aprendizaje y estilo cognitivo. Revista Suma Psicológica, 19(2), 39-50.

Martín, M., Bueno, J., \& Ramírez, M. (2010). Evaluación del aprendizaje autorregulado en estudiantes de Bachillerato mexicanos. Revista Aula Abierta, 38(1), 59-70.

Monereo, C. (2001). La enseñanza estratégica. Enseñar para la autonomía. Aula de Innovación, 100, 6-10.

Multon, K., \& Brown, S. (1991). Relation of self effcacy beliefs to academic outcomes: a metaanalytic investigation. Journal of Counseling Psychology, 38, 30-38.

Nelson, T. O., Narens, L. (1990). Metamemory: a theoretical framework and new findings. En G. H. Bower (Ed.) The psychology of learning and motivation (pp. 125-173). New York: Academic Press.

Nelson, T. O. (1992). Metacognition: Core Readings. Boston: Allyn and Bacon.

Nocito, G. (2013). Autorregulación del aprendizaje de alumnos de grado. Universidad Complutense de Madrid. Recuperado de http://eprints.ucm.es/24036/1/T35049.pdf

Núñez, J., González-Pienda, J., \& Rosario, P. (2006). Evaluación de los procesos de autorregulación mediante el autoinforme. Psicothema, 18(3), 353-358. 
Panadero, E. (2017). A Review of Self-regulated Learning: Six Models and Four Directions for Research. Front. Psychol., 8(1-28). Doi: 10.3389/fpsyg.2017.00422

Peña- Ayala, A., Cárdenas, L (2015). A conceptual model of the metacognitive activity. En A. Peña (Ed.) Metacognition: Fundaments, applications, and trends (pp. 39-64). New York: Springer international.

Pierce, S. H., \& Lange, G. (2000). Relationships among metamemory, motivation and memory performance in young school-age children. British Journal of Developmental Psychology, $18,121-135$.

Pintrich, P., De Groot, E. (1990). Motivational and self regulated learning components of classroom academic performance. Journal of Educational Psychology, 82(1), 33-40.

Pintrich, P. R., Smith, D. A., García, T. \& McKeachie, W. J. (1991). A manual for the use of the Motivated Strategies for Learning Questionnaire (MSQL). Ann Arbor, MI: NCRIPTAL: The University of Michigan.

Pintrich, P., Smith, D., García, T., \& McKeachie, W. (1993). Reliability and predictive validity of the motivated strategies for learning questionnaire (MSQL). Educational and Psychological Measurement, 53, 801-803.

Pintrich, P. (2004). A conceptual framework for assessing motivation and self-regulated learning in college students. Educational Psychology Review, 16, 385-407.

Ramírez, M. (2016). Modelo causal de los factores asociados al aprendizaje autorregulado como mediador del rendimiento académico en estudiantes universitarios. (Tesis Doctoral, Universidad Complutense de Madrid, Madrid, España). Recuperado de file:///Users/luzestela/Desktop/DIANA\%20/Modelo\%20causal\%20de\%20los\%20factores\%20asociados \%20 al\%20aprendizaje $\% 20$ autorregulado $\% 20$ como $\% 20$ mediador $\% 20$ del $\% 20$ rendimiento\%20a.webarchive

Ramírez, M., Canto, J., Bueno, J., \& Echazarreta, A. (2013). Validación psicométrica del Motivated Strategies for Learning Questionnaire en universitarios mexicanos. Electronic Journal of Research in Educational Psychology, 11(1), 193-214.

Rothman, A., Baldwin, A., Hertel, A., \& Fuglestad, P. (2011). Self-regulation and behavior change: Disentangling behavioral initiation and behavioral maintenance. In K. Vohs, \& R. Baumeister. Handbook of self-regulation. Research, Theory, and Applications (pp. 106-122). Nueva York: Guilford Publications. 
Sawyer, K. (2014). The Cambridge Handbook of the Learning Sciences. New York: Cambridge University Press.

Sheskin, D. (2007). Handbook of parametric and nonparametric statistical procedures. Boca Ratón, FL: Ed. Chapman \& Hall/crc.

Schraw, G., \& Gutiérrez, A. (2015). Metacognitive strategy instruction that highlinghts the role of monitoring and control processes. In A. Peña (2015). Metacognition: Fundaments, applications, and trends (pp. 3-15). Nueva York: Springer international.

Schunk, D. (2012). Teorías del aprendizaje. Una perspectiva educativa. México D.F.: Editorial Pearson.

Schunk, D., \& Zimmerman, B. (1994). Self-regulation in education: retrospect and prospect $305-$ 314. En Autor, Self-regulation of learning and performance. Issues and educational applications. Hillsdale, NJ: Erlbaum.

Suarez, J. M., \& Fernandez, A. P. (2011). A model of how motivational strategies related to the expectative component affect cognitive and metacognitive strategies. Electronic Journal of Research in Educational Psychology, 9(2), 641-658.

Suárez, J., Fernández, A., Sánchez, V., \& Zamora, A. (2016). Incidencia de las estrategias motivacionales de valor sobre las estrategias cognitivas y metacognitivas en estudiantes de secundaria. Revista Complutense de Educación, 27(2), 421-435.

Tarricone, P. (2011). The taxonomy of metacognition. Nueva York: Psychology Press.

Winne, P., Azevedo, R. (2014). Metacognition. In K. Sawyer, The Cambridge handbook of the learning sciences (pp. 63-87). New York: Cambridge University Press.

Wolters, C. (1998). Self-regulated learning and college student's regulation of motivation. Journal of Educational Psychology, 90, 224-235.

Zimmerman, B. (1989). A social cognitive view of self-regulated academic learning. Journal of Educational Psychology, 81(3), 329-339.

Zimmerman, B. (2008). Investigating self-regulation and motivation: Historical background, methodological developments, and future prospects. American Educational Research Journal, 45, 166-183.

Zimmerman, B., \& Schunk, D. (1989). Self-regulated learning and academic achievement. Theory, research and practice. NY: Springer-Verlag. 